\title{
Verbraucherorganisationen und Verbraucherpolitik als Intermediäre der Nachhaltigkeit. Eine Analyse der institutionellen und organisationalen Einbettung nachhaltigen Konsums
}

\author{
Sebastian Nessel
}

Online publiziert: 17. Januar 2017

(C) Der/die Autor(en) 2016. Dieser Artikel ist eine Open-Access-Publikation.

Zusammenfassung Dieser Beitrag greift aktuelle Erklärungsansätze auf, die nachhaltigen Konsum als eine sozial und institutionell eingebettete Praxis verstehen. Die deutschen Verbraucherorganisationen und die staatliche Verbraucherpolitik werden als ein zentraler Bestandteil der institutionellen und organisationalen Einbettung des nachhaltigen Konsums kenntlich gemacht. Der Aufsatz arbeitet systematisch die Strategien dieser verbraucherpolitischen Akteure heraus, um zu verdeutlichen, wie das soziale und organisationale Umfeld von Konsumenten und Anbietern auf nachhaltige Konsummuster einwirkt. Die Analyse zeigt, dass verbraucherpolitische Organisationen zentrale Intermediäre nachhaltigen Konsums darstellen, die die Handlungsabsichten, die Einstellungen, das Wissen sowie die Koordination von Akteuren im Bereich nachhaltiger Märkte beeinflussen.

Schlüsselwörter Nachhaltigkeit · Konsumsoziologie · Wirtschaftssoziologie · Verbraucherorganisationen · Verbraucherpolitik

\section{Consumer organizations and consumer policy as intermediaries of sustainability. An analysis of the institutional and organizational embeddedness of sustainable consumption}

\footnotetext{
Abstract Current studies have demonstrated that sustainable consumption is influenced by the social and institutional embeddedness of actors. In this article German consumer organizations and German consumer policy are defined as being substantial parts of the institutional and organizational embeddedness of consumers and firms. The strategies of these consumer policy organizations are analyzed system-

S. Nessel $(\bowtie)$

Institut für Soziologie, Karl-Franzens-Universität Graz, Universitätsstraße 15/G3, 8010 Graz,

Österreich

E-Mail: sebastian.nessel@uni-graz.at
} 
atically. These analyses show that consumer organizations and consumer policy are central intermediaries of sustainable consumption and influence the motivations, the values, the knowledge and the coordination of actors in the market for sustainable products.

Keywords Sustainability - Sociology of consumption · Economic sociology • Consumer organisations

\section{Les associations de consommateurs et la politique de la consommation comme intermédiaires du développement durable. Une analyse de l'encadrement institutionnel et organisationnel de la consommation durable}

Résumé Cet article fait écho aux approches théoriques qui conçoivent la consommation durable comme une pratique socialement et institutionnellement encadrée. Les associations de consommateurs allemandes et la politique étatique de la consommation sont désignées comme un élément essentiel de l'encadrement institutionnel et organisationnel de la consommation durable. Cet article examine de manière systématique les stratégies de ces acteurs de la politique de la consommation pour mettre en évidence comment l'environnement social et organisationnel des consommateurs et des fournisseurs influe sur les modes de consommation durable. Cette analyse montre que les organisations de la politique de la consommation constituent des intermédiaires essentiels de la consommation durable qui exercent une influence sur les intentions, les attitudes, l'information et la coordination des acteurs du marché des biens de consommation durables.

Mots-clés Développement durable · Sociologie de la consommation · Sociologie économique $\cdot$ Associations de consommateurs · Politique de la consommation

\section{Einleitung}

Die Rolle von Konsumenten wurde in der deutschen Politik lange Zeit ausschließlich ökonomisch verstanden. Insbesondere die Marktstellung der Konsumenten gegenüber Anbietern, ihre ,rationalen“ Kaufentscheidungen und ihr Beitrag zu ,funktionierenden“ Märkten bildeten bis in die 1980er-Jahre die zentralen Ansatzpunkte für verbraucherpolitische Maßnahmen (Mitropoulos 1997, S. 18 ff.; Nessel 2016, S. 77 ff.). Vor dem Hintergrund einer verstärkten öffentlichen Wahrnehmung globaler Umweltprobleme haben seit Mitte der 1980er-Jahre zunächst Verbraucherorganisationen Konsumenten und Unternehmen als „,sozial verantwortliche“ Marktteilnehmer definiert und Nachhaltigkeit als übergreifendes Ziel der Verbraucherpolitik aufgefasst. Im Zuge der globalen Nachhaltigkeitsdebatte, eingeleitet u. a. durch den Brundtland-Bericht 1987 und verstärkt durch die Rio-Konferenz 1992, hat auch die staatliche Verbraucherpolitik die sozial-ökologische Verantwortung von Marktakteuren aufgegriffen. Die Förderung nachhaltiger Verhaltensweisen wurde durch die nationale Nachhaltigkeitsstrategie 2002 und den Aktionsplan Verbraucherschutz 
2003 dann auch institutionell als Ziel des politischen Handelns verankert. Vor dem Hintergrund des verbraucherpolitischen Leitbildes mündiger und verantwortungsvoller Konsumenten und Firmen (Belz und Billharz 2007; Müller 2001) versuchen verbraucherpolitische Akteure seitdem, durch Öffentlichkeitsarbeit, Ratgeber oder Produktbewertungen die institutionellen und kulturellen Rahmenbedingungen nachhaltigen Konsums zu beeinflussen.

Die Bedeutung verbraucherpolitischer Akteure für nachhaltigen Konsum ist bislang jedoch kaum systematisch erforscht worden. Zwar zeichnet sich in der jüngeren Diskussion immer deutlicher ab, dass ein nachhaltiger, politischer bzw. ethischer Konsum $^{1}$ von Verbrauchern nicht nur auf individuelle Faktoren wie Bildung, Geschlecht oder Einkommen zurückgeführt werden kann, sondern ganz wesentlich durch das soziale Umfeld der Akteure beeinflusst wird, z. B. durch Haushaltskontexte (Matthies und Wallis 2015), soziale Bewegungen (Micheletti und Stolle 2007), staatliche Labels (Koos 2011) oder das Wohlstandsniveau eines Landes (Zhang 2015). Erste Arbeiten weisen auch darauf hin, dass verbraucherpolitische Akteure nachhaltigen Konsum beeinflussen können, indem sie Konsumenten glaubhafte Produktinformationen zur Verfügung stellen (Fricke und Schrader 2014). Eine umfassende Analyse verbraucherpolitischer Akteure als Teil des institutionellen und organisationalen Umfelds von Akteuren und ihrer Bedeutung für nachhaltigen Konsum steht bisher jedoch noch aus. Speziell die Fragen, wie verbraucherpolitische Akteure nachhaltige Konsummuster beeinflussen und welche Strategien sie hierzu anwenden, wurden bisher nur unzureichend beantwortet.

An diesen Fragen und an der sozialen Einbettung nachhaltigen Konsums setzt der vorliegende Beitrag an. Es wird argumentiert, dass verbraucherpolitische Akteure zentrale Intermediäre der Nachhaltigkeit sind, die sowohl nachhaltige Konsumals auch Produktionsmuster beeinflussen. Um diese These zu begründen, geht der Beitrag in fünf Schritten vor. Zunächst wird ein analytischer Rahmen ausgearbeitet, der vier zentrale Einflussfaktoren nachhaltigen Konsums unterscheidet (Abschn. 2): die Absicht von Akteuren, nachhaltige Produkte zu kaufen (Motivationsebene); ihre Einstellungen gegenüber Umwelt und Gesellschaft (Einstellungsebene); ihr Wissen über nachhaltige Produkte im Speziellen und über Nachhaltigkeit im Allgemeinen (Wissensebene); sowie die Koordination von Konsumenten und Anbietern (Koordinationsebene). Daran anschließend werden die Strategien von Verbraucherorganisationen (Abschn. 3) und der Verbraucherpolitik (Abschn. 4) herausgearbeitet und hinsichtlich ihres Beitrages für nachhaltige Konsummuster untersucht. Abschließend wird verdeutlicht, dass verbraucherpolitische Akteure die Motivationen, die Einstellungen, das Wissen sowie die Koordination von Anbietern und Konsumenten auf nachhaltigen Märkten beeinflussen (Abschn. 5). Nachhaltiger Konsum wird so als eine durch Organisationen vermittelte soziale Praxis sichtbar gemacht.

\footnotetext{
${ }^{1}$ Ich verwende diese Begriffe und die damit verbundenen Konzepte im Folgenden synonym für die intentionale Bestrebung von Akteuren, durch Konsumhandlungen politische Ziele im Sinne der Nachhaltigkeit zu verfolgen (wie Umweltschutz oder eine inter- und intragenerationale Gerechtigkeit; vgl. WCED 1987). Zur Wesensverwandtschaft dieser Begriffe und zur Konzeption des nachhaltigen Konsums vgl. Heidbrink und Schmidt 2011, S. 31 ff., und Micheletti und Stolle 2012.
} 


\section{Motivationen, Einstellungen, Wissen und Koordination als Voraussetzungen nachhaltiger Konsummuster}

Im nachfolgenden Abschnitt wird ein analytischer Rahmen zur Analyse nachhaltiger Konsummuster skizziert. Vier miteinander verwobene Einflussfaktoren auf eine nachhaltige Konsumweise werden unterschieden: die Absicht, nachhaltige Produkte zu kaufen (Motivationsebene); die Einstellung gegenüber Umwelt und Gesellschaft (Einstellungsebene); das Wissen über nachhaltige Produkte und über Nachhaltigkeit (Wissensebene); sowie die Koordination von Konsumenten und Anbietern (Koordinationsebene).

Die Motivationsebene verweist auf die mit dem Kauf oder der Herstellung nachhaltiger Produkte verbundenen Handlungsabsichten. Die Handlungsabsichten von Akteuren können eher wert- oder eher zweckrational begründet werden. Mit dem Kauf nachhaltiger Produkte kann die Absicht verbunden sein, die natürliche Umwelt und die Lebensbedingungen der heutigen und zukünftigen Generationen zu schonen (Joshi und Rahman 2015; Steg 2015). Eine solche Absicht korrespondiert dann weitgehend mit der verbreiteten wissenschaftlichen und politischen Vorstellung von Nachhaltigkeit, die im Brundtland-Bericht (WCED 1987) definiert wurde. Konkret bedeutet dies, dass mögliche Folgen des Konsums nicht nur im Hinblick auf das eigene Selbst, sondern auch mit Blick auf dessen Umwelt- und Sozialverträglichkeit abgewogen werden. Solche Absichten sind eher am wertrationalen Pol von Handlungsmotiven zu verorten und als sozial-ökologische Handlungsabsichten zu kennzeichnen. Der Kauf nachhaltiger Produkte kann aber auch damit begründet werden, sozialen Status auszudrücken (Baumann et al. 2015, S. 419; Welsch und Kühling 2009, S. 173) oder gesünder leben zu wollen (Lorenz 2006; Magnusson et al. 2003). Solche Handlungsabsichten sind eher dem zweckrationalen Pol von Handlungsmotiven zuzuordnen. Wie Yatish Joshi und Zillur Rahman (2015) in einer Meta-Analyse vorliegender Untersuchungen zeigen, sind sowohl zweck- als auch wertrationale Motive bedeutsam, um den Kauf nachhaltiger Produkte nachzuvollziehen (vgl. auch Magnusson et al. 2003). ${ }^{2}$

Die Motivationsebene ist eng verknüpft mit der Einstellungsebene. Folgt man der sozialpsychologischen Umweltforschung, dann korrespondieren sozial-ökologische Absichten zum Kauf nachhaltiger Produkte vor allem mit „,biospheric“ und ,,altruistic values“, d.h. mit dem Einbezug der Umwelt oder moralisch einzubeziehender (imaginierter) Anderer in den eigenen Entscheidungshorizont, zweckrationale Motivationen hingehen mit ,hedonistic“ oder „,egoistic values“, die eher das eigene Erleben oder den eigenen Nutzen von Handlungen ausdrücken (Steg 2015). Wie in zahlreichen Studien gezeigt wurde, beeinflussen insbesondere sozial-ökologische Einstellungen nachhaltige Produktkäufe (Steg 2015; Thøgersen und Ölander 2002; Umweltbundesamt 2014, S. 68 ff.). Positive Einstellungen gegenüber der Umwelt und gegenüber Anderen tragen dazu bei, dass der Preis eines Produktes weniger

\footnotetext{
2 Wie Welsch und Kühling (2009) verdeutlichen, variiert der Einfluss von zweck- und wertrationalen Motiven auf Handlungsentscheidungen je nach Marktfeld: Für den Kauf von Bio-Lebensmitteln stellen die Autoren fest, dass insbesondere sozial-ökologische Motivationen eine Rolle spielen, beim Kauf von Solaranlagen hingegen eher Statusmotive (,Mercedes-Benz on the rooftop“; ebd., S. 172).
} 
gewichtet wird und Konsumenten auch einen Preisaufschlag für nachhaltige Angebote akzeptieren (Tanner und Kast 2003, S. 893). Auch scheinen sozial-ökologisch eingestellte Konsumenten die Auswirkungen des eigenen Konsums auf Andere oder die Umwelt eher in ihren Handlungsentscheidungen zu berücksichtigen (Steg 2015, S. $75 \mathrm{ff}$.) und politische Maßnahmen zur Förderung von Nachhaltigkeit eher zu akzeptieren (Thøgersen und Ölander 2002, S. 623 ff.). Die Förderung sozial-ökologischer Motivationen und Einstellungen wird daher als ein wichtiger Ansatzpunkt politischer Maßnahmen zur Förderung nachhaltigen Konsums gesehen (Schrader et al. 2013; Thøgersen 2005).

Für die weiteren Ausführungen ist nun bedeutsam, dass Einstellungen und Handlungsabsichten nicht als gegeben vorausgesetzt werden können, sondern ein Wissen über Nachhaltigkeit und über nachhaltige Produkteigenschaften voraussetzen. Ein solches Wissen wird ganz wesentlich durch das soziale Umfeld von Akteuren produziert und vermittelt - so z. B. durch soziale Bewegungen (Balsiger 2010; Micheletti und Stolle 2007), durch Haushaltskontexte (Matthies und Wallis 2015), durch schulische und außerschulische Bildung (Thøgersen 2005, S. 156 f.), wissenschaftliche Studien (Steg 2015) oder, wie im Folgenden gezeigt wird, durch verbraucherpolitische Akteure. Um die Wissensebene nachhaltigen Konsums zu analysieren, wird im Folgenden an die Verbraucherbildungsforschung angeschlossen, die verschiedene verbraucherrelevante Wissensbestände differenziert und die ökonomische Grundbildung von einem spezifischen Produktwissen abgrenzt (Remmele 2012). Diese Diskussion aufgreifend, werden in diesem Beitrag zwei Wissensbestände unterschieden, die beide wichtige Voraussetzungen nachhaltigen Konsums darstellen: ein allgemeines Wissen über Nachhaltigkeit sowie über nachhaltige Produktkennzeichnungen (Nachhaltigkeitswissen) sowie ein spezifisches Wissen zur Einschätzung nachhaltiger Produktqualitäten (Produktwissen). Nachhaltigkeitswissen meint ein Grundverständnis über die Effekte von Konsum und Produktion auf Umwelt und Gesellschaft sowie ein Problembewusstsein über den Beitrag einzelner Handlungen für eine nachhaltige Entwicklung. Darüber hinaus beinhaltet ein solches Wissen ein allgemeines Verständnis sozial-ökologischer Produkteigenschaften sowie ihrer Kennzeichnungen. Ein solches Wissen ist einerseits zentral für die Entstehung sozial-ökologischer Handlungsabsichten und Einstellungen, denn diese setzen implizit voraus, dass Nachhaltigkeit als soziales Problem wahrgenommen wird und Akteure ihre eigenen Konsumhandlungen mit deren möglichen Folgen für Umwelt und Gesellschaft verbinden (Micheletti und Stolle 2007). Andererseits ist Nachhaltigkeitswissen eine Voraussetzung auch für zweckrationale Handlungsabsichten. Dass Akteure z. B. davon ausgehen, dass „Bio-Produkte“ besser für die eigene Gesundheit seien, beinhaltet, dass sie ein allgemeines Verständnis über die Eigenschaften dieser Produkte (z. B. ihrer Anbaumethoden) haben. Darüber hinaus müssen sowohl sozial-ökologisch als auch zweckrational eingestellte und motivierte Konsumenten die Kennzeichnungen nachhaltiger Produkte kennen - und anerkennen (vgl. unten). Denn ohne ein Wissen über Funktion und Inhalt solcher Kennzeichnungen werden Konsumenten nachhaltige Angebote in ihren Kaufentscheidungen kaum berücksichtigen (Thøgersen 2005, S. 158 f.).

Allerdings führen nachhaltigkeitsrelevante Handlungsabsichten und Einstellungen nicht automatisch zu nachhaltigen Produktkäufen. In vielen Studien wurde da- 
rauf aufmerksam gemacht, dass insbesondere sozial-ökologische Motivationen bzw. Einstellungen oftmals nicht zu den entsprechenden Handlungsentscheidungen führen (,value-action gap“ bzw. „motivation-behavior gap“; vgl. Young et al. 2010, S. 22). Als Gründe hierfür werden u. a. Ziel- und Wertkonflikte (Thøgersen und Ölander 2002, S. 626), eingespielte Routinen (Steg 2015) oder Zeitbarrieren (Tanner und Kast 2003, S. 893 ff.) genannt. Ein zentraler Faktor zur Überwindung dieser „Barrieren" und zum Kauf nachhaltiger Produkte ist der Abbau von Unsicherheit über deren sozial-ökologische Qualitätseigenschaften (Fricke und Schrader 2014; Young et al. 2010, S. 22 ff.). Eine solche Unsicherheit ergibt sich daraus, dass nachhaltige Qualitäten von Produkten für die Konsumenten selbst kaum einschätzbar sind. Ihr Preis gibt keine Auskunft darüber, ob sie Umwelt und Gesellschaft im Herstellungsprozess mehr oder weniger belasten. Auch können nachhaltige Qualitätseigenschaften ,,weder durch Inspektion vor dem Kauf noch durch eigene Konsumerfahrung“ direkt bewertet werden (Fricke und Schrader 2014, S. 207). Märkte für nachhaltige Güter können daher im Anschluss an den französischen Wirtschaftssoziologen Lucien Karpik (2010) als „Märkte des Besonderen“ gekennzeichnet werden, auf denen „Instanzen der Urteilsbildung“ (Produktkennzeichnungen, Expertenmeinungen, Rankings und Ratgeber) Konsumenten wichtige Hinweise zur Einschätzung von Produktqualitäten bieten (ebd., S. 62 ff.). Um die Unsicherheit über nachhaltige Produktqualitäten abzubauen, können Konsumenten auf eine Vielzahl solcher Urteilsinstanzen zurückgreifen. Zu nennen sind etwa sozial-ökologische Labels (Koos 2011; Stolle und Micheletti 2013, S. 137 ff.), wissenschaftliche Studien (Steg 2015, S. 85) oder Produktbewertungen verbraucherpolitischer Akteure (Fricke und Schrader 2014) und NGOs (Münch 2008). Insbesondere für staatliche Labels wurde empirisch gezeigt, dass sie Produktkäufe stark beeinflussen (Koos 2011; Stolle und Micheletti 2013, S. 137 ff.; Umweltbundesamt 2014, Kap. 7). Durch ihre Bewertungen tragen die genannten Instanzen dazu bei, nachhaltige Produkte zu klassifizieren und Konsumenten ihre Auswahl zu erleichtern. Allerdings müssen Konsumenten diese Produkturteile und Klassifikationen kennen, zwischen verschiedenen Bewertungen auswählen und ihnen auch vertrauen. Die Darstellung der Wissensebene zusammenfassend, lässt sich an dieser Stelle zunächst festhalten, dass das Nachhaltigkeitswissen eine Voraussetzung für die Entstehung oder Bekräftigung nachhaltigkeitsrelevanter Handlungsabsichten und Einstellungen ist und so indirekt auf nachhaltigen Konsum einwirkt, während das Produktwissen eher direkt die Auswahl nachhaltiger Produkte beeinflusst und die Umsetzung von Handlungsabsichten in Produktkäufe begünstigt.

Als vierter Einflussfaktor nachhaltigen Konsums ist schließlich die in der Literatur bisher weitgehend unberücksichtigte Koordination zwischen Marktakteuren in den Blick zu nehmen. Die Koordinationsebene verweist darauf, dass Konsumenten und Anbieter ihre Handlungsabsichten und -entscheidungen auf Märkten nur zusammen mit Anderen umsetzen können. Anbieter und Nachfrager müssen letztlich zusammengeführt werden, damit ein nachhaltiger Konsum realisiert werden kann. Eine solche Koordination von Akteuren kann durch gleichgerichtete Handlungsabsichten begünstigt werden (Bourdieu 2005). Nachhaltig motivierte Konsumenten und Firmen werden demnach eher zusammenfinden als nachhaltig orientierte Verbraucher und weniger nachhaltige Anbieter. Folgt man zentralen Einsichten der 
neueren Wirtschaftssoziologie, dann ist die Koordination von Akteuren darüber hinaus an die Reduktion von Unsicherheit gebunden (Beckert 2007). Damit Akteure Handlungsabsichten umsetzen können, müssen sie demnach ihre Erwartungen über die Absichten Anderer sowie deren Anforderungen an ihr eigenes Handeln stabilisieren. Eine dauerhafte Koordination zwischen Marktakteuren setzt somit voraus, dass Firmen die Erwartungen von Konsumenten an ihre Produkte einschätzen und begründet annehmen können, dass diese eine Zahlungsbereitschaft für sozial-ökologische Produktqualitäten haben. Konsumenten müssen hingegen von der Qualität dieser Produkte überzeugt sein. Um ihre Erwartungen über Produkteigenschaften zu stabilisieren, können Konsumenten, wie bereits erwähnt, auf Produktbewertungen von Instanzen der Urteilsbildung zurückgreifen. Um die Zahlungsbereitschaft von Konsumenten und ihre Erwartungen einzuschätzen, können Anbieter hingegen Marktforschung betreiben oder ihre Konkurrenten (White 1981) sowie andere Akteure ihrer sozialen Umwelt wie z. B. Verbraucherorganisationen (Nessel 2015) oder NGOs (Bluhm 2008) beobachten. Diese Überlegungen verweisen darauf, dass die Übereinstimmung von Erwartungen eine zentrale Voraussetzung für die Koordination von Marktakteuren ist. Auf der Anbieterseite ist darüber hinaus bedeutsam, dass nachhaltige Produkteigenschaften an Konsumenten glaubhaft vermittelt werden. Allerdings werden die Produktversprechen der Anbieter von Konsumenten oftmals infrage gestellt, sofern sie nicht durch anbieterunabhängige Organisationen glaubhaft bescheinigt werden (Fricke und Schrader 2014; Münch 2008). Das Vertrauen in die jeweils andere Marktseite bildet schließlich eine weitere Voraussetzung zur Koordination auf nachhaltigen Märkten. Wie zu zeigen sein wird, können verbraucherpolitische Akteure ein solches Vertrauen herstellen, aber auch in Zweifel ziehen.

Die vorangegangenen Ausführungen zusammenfassend, kann festgehalten werden, dass Handlungsabsichten, Einstellungen, Nachhaltigkeits- und Produktwissen sowie die Koordination von Akteuren zentrale Einflussfaktoren nachhaltigen Konsums sind. Wie verdeutlicht, werden diese Faktoren maßgeblich durch das soziale Umfeld von Akteuren beeinflusst. Um die Bedeutung des sozialen Umfeldes für den nachhaltigen Konsum genauer zu analysieren, werden im Folgenden die Strategien verbraucherpolitischer Akteure herausgearbeitet und hinsichtlich ihrer Bedeutung für sozial-ökologische Verhaltensweisen untersucht.

\section{Verbraucherorganisationen und Nachhaltigkeit}

Verbraucherorganisationen verfolgen den Anspruch, Konsumenten zu organisieren, zu vertreten und zu beraten (Nessel 2016). Bis in die 1980er-Jahre standen vor allem die Verbesserung der Marktstellung des Konsumenten gegenüber Firmen und ihre politische Vertretung im Fokus deutscher Verbraucherorganisationen. Seit Mitte der 1980er-Jahre wurden dann auch die ökologischen und gesellschaftlichen Probleme des Konsums sowie die Verantwortung von Konsumenten und Anbietern hierfür aufgegriffen (Müller 2001, S. 8). Gegenwärtig ist die gesellschaftliche Verankerung von Nachhaltigkeit das Hauptziel zweier privater Verbraucherorganisationen: der Verbraucherinitiative (VI), die ca. 8000 individuelle Mitglieder und ca. 176 Organisationen aus der Zivilgesellschaft umfasst, sowie der 2007 gegründeten Organisation 
Utopia, deren Internetangebote monatlich ca. 80.000 Mal abgerufen werden und die Firmen zur Nachhaltigkeit berät. Auch staatlich finanzierte Verbraucherorganisationen in Deutschland wie die Stiftung Warentest (STW), die Verbraucherzentrale Bundesverband (vzbv) und die Verbraucherzentralen der Länder (Vz) haben inzwischen die Förderung sozial-ökologischer Verhaltensweisen als weiteres Ziel ihrer Arbeit verankert. ${ }^{3}$ Um Konsum- und Produktionsmuster $\mathrm{zu}$ beeinflussen, wenden Verbraucherorganisationen im Wesentlichen drei Strategien an: die Verbreitung von Informationen, in denen das Nachhaltigkeitskonzept beschrieben und konkretisiert wird (allgemeine Verbraucherinformation), die Beurteilung nachhaltiger Produktqualitäten (spezifische Verbraucherinformation) sowie Beratungsangebote für Firmen (Unternehmensdialoge). Diese Strategien werden im Folgenden anhand exemplarischer Praktiken verdeutlicht und im Hinblick auf einen nachhaltigen Konsum analysiert.

Die Strategie der allgemeinen Verbraucherinformation wird durch vielfältige Praktiken umgesetzt. In Publikationen und auf Internetseiten wird am Beispiel einzelner Marktfelder (Kosmetik, Finanzanlagen, Lebensmittel u. v. m.) verdeutlicht, was Nachhaltigkeit bedeuten kann und dass nachhaltige Verhaltensweisen im Alltag u. a. durch den Kauf sozial-ökologischer Produkte oder durch eine Reduktion des Konsums (z. B. Energiesparen) umgesetzt werden können. Eine ähnliche Vorgehensweise findet sich in schulischen und außerschulischen Bildungsprojekten (Vz, vzbv) sowie in Angeboten zur Erwachsenenbildung (Vz, VI), in denen nachhaltige Konsumkompetenzen vermittelt werden. Schließlich verleihen Verbraucherorganisationen auch Preise für ein nachhaltiges Engagement. Utopia vergibt den „Utopia Award“ und den „Changemaker-Titel“, die Deutsche Stiftung Verbraucherschutz, eine Tochterorganisation der vzbv, den ,Bundespreis Verbraucherschutz“, und die VI prämiert im Projekt „Nachhaltig einkaufen“ Hersteller mit Gold, Silber und Bronze für ihr nachhaltiges Engagement. Die Benennung solcher Best-Practice-Beispiele folgt der Idee, Anbieter und Konsumenten durch positive Anreize zu nachhaltigen Verhaltensweisen zu motivieren.

Am Beispiel des Projekts „öko-fair“ der VI kann die Strategie der allgemeinen Verbraucherinformation exemplarisch verdeutlicht werden. Im Rahmen von „ökofair" wird auf einer Internetseite über Nachhaltigkeitszusammenhänge berichtet. Für zahlreiche Marktbereiche werden Produktmerkmale (Herkunft, Vorkommen, Anbau), sozial-ökologische Qualitätsdimensionen (Bio-Zertifizierung, Fairer Handel) sowie nachhaltige Handlungsstrategien dargestellt: „Das reicht von der Beschreibung verschiedener Produkte und ihrer öko-fairen Alternativen [Bereich, clever konsumieren'], über den richtigen Umgang mit Abfall oder Nahrungsmitteln bis hin zu Themen wie Umwelt- und Sozialverantwortung [Bereich ,verantwortlich handeln '].“ (VI 2012, S. 10) Die dargestellten Informationen sollen einerseits ein Verständnis über Nachhaltigkeit fördern, indem Herstellungsbedingungen und Zusammenhänge zwischen Produktion, Konsum und nachhaltiger Entwicklung veranschaulicht werden. Andererseits sollen diese „Entscheidungshilfen“ den Konsum in Richtung Nachhaltigkeit bewegen und eine „,bewusste Kaufentscheidung für öko-faire Pro-

\footnotetext{
3 Vgl. zu den Organisationsstrukturen, Mitgliedern und Strategien der genannten Organisationen ausführ-
} lich Nessel 2016, S. 129 ff. 
dukte“ anleiten. ${ }^{4}$ Die Beiträge auf der Homepage werden ca. 75.000 Mal pro Monat abgerufen. Eine breitere Öffentlichkeit erreichen diese Informationen durch Pressemitteilungen, monatliche Newsletter sowie eine „Facebook-Fanseite“. Um nachhaltigen Konsum zu fördern, richtet sich das Projekt auch an Firmen und NGOs, die ausgewählte Nachhaltigkeitsaktivitäten präsentieren können.

Durch die genannten Praktiken zielen die Verbraucherorganisationen darauf ab, Nachhaltigkeit für Akteure greifbar zu machen und auf die problematischen Effekte von Konsum und Produktion auf Umwelt und Gesellschaft(en) hinzuweisen. Um die so definierten konsum- und produktionsinduzierten sozial-ökologischen Probleme abzumildern, verweisen sie zugleich auf mögliche Lösungsstrategien wie den Kauf nachhaltiger Produkte oder die Reduktion von Konsum. Der Verweis auf andere Konsumenten und Organisationen, die bereits nachhaltig konsumieren oder produzieren, soll hingegen verdeutlichen, dass die angesprochenen Akteure nicht allein für Nachhaltigkeit eintreten sollen. Da der Einzelne kaum etwas dazu beitragen kann, die globalen Umweltprobleme zu verringern, ist ein Wissen über die Handlungsbereitschaft Anderer wichtig, um Akteure zu motivieren, auch selbst einen Beitrag zur Nachhaltigkeit zu leisten (Thøgersen 2005, S. 145). Über die Strategie der allgemeinen Verbraucherformation werden sozial-ökologische Verhaltensweisen schließlich als gesellschaftlich wünschenswert definiert und das Leitbild mündiger und verantwortungsvoller Konsumenten und Anbieter in der Öffentlichkeit propagiert. Diese Strategie folgt somit der Idee, dass ein entsprechendes Wissen über Nachhaltigkeit sowie ein Problembewusstsein für den Effekt einzelner Handlungen auf Umwelt und Gesellschaft wichtige Voraussetzungen für die Entwicklung sozialökologischer Handlungsabsichten und Einstellungen sind.

Eine zweite zentrale Strategie von Verbraucherorganisationen ist die spezifische Verbraucherinformation. Im Rahmen dieser Strategie wird eine vergleichende Beurteilung der Nachhaltigkeit von Produkten und ihrer Qualitätseigenschaften vorgenommen. Die Stiftung Warentest führt dazu Corporate-Social-Responsibility-Tests durch (STW 2004). In diesen CSR-Tests werden sozial-ökologische Produkte abgebildet und je nach ihrem Nachhaltigkeitspotenzial im Schulnotensystem von ,sehr gut“ bis „mangelhaft“ bewertet. Die Testergebnisse können dann kostenpflichtig in Zeitschriften (test und Finanztest) und auf einer Homepage (test.de) abgerufen werden. Eine weitere Verbreitung finden diese Ergebnisse über die Hersteller, die damit werben (Schrader 2008). Darüber hinaus berichten auch die Medien über ausgewählte Testergebnisse. Die VI bewertet Produktqualitäten in öffentlich finanzierten Projekten und verbreitet ihre Bewertungen vor allem über Internetseiten (label-online.de, nachhaltig-einkaufen.de, öko-fair.de). Hervorzuheben ist, dass im Projekt „Label-online“ auch die Labels selbst bewertet werden. Ausgangspunkt dieses Projekts ist die plausible Feststellung, dass Labels glaubhafte Informationen über die Qualität von Produkten bereitstellen können. Als problematisch angesehen wird aber, dass in Deutschland mittlerweile mehr als tausend davon existieren und sie dadurch ihre Orientierungsfunktion für Konsumenten einbüßen (VI 2012, S. 10). Dementsprechend fordert auch ein Expertengutachten des ehemaligen Bundesministeriums für Ernährung, Landwirtschaft und Verbraucherschutz (BMELV) eine ,,weitere Re-

\footnotetext{
4 Vgl. http://www.oeko-fair.de/wir-ueber-uns/oeko-fairde. Zugegriffen: Okt. 2016.
} 
duzierung des Label-,Wildwuchses', der eher Konsumentenverwirrung als Entlastung und Orientierung schafft" (Reisch 2003, S. 29; vgl. auch Schrader et al. 2013, S. 11). Um die Unübersichtlichkeit zertifizierter Produkte abzubauen, vergleicht die VI verschiedene Standardisierungen und macht deren Kriterien deutlich. Soziale (z. B. „Fair-Trade“) und ökologische Labels (z. B. „Bio-Siegel“) sowie nachhaltige Managementstandards (z. B. „ISO 14000“) werden detailliert beschrieben und bewertet (besonders empfehlenswert; empfehlenswert; eingeschränkt empfehlenswert; nicht empfehlenswert). Die Ergebnisse werden dann über Internetseiten, Newsletter und Presseinformationen verbreitet. In Bezug auf das Portal Utopia ist schließlich hervorzuheben, dass angemeldete Nutzer der Homepage einbezogen werden, um nachhaltige Produkte zu unterscheiden, und diese auch das wahrgenommene PreisLeistungsverhältnis sowie die funktionalen Güterqualitäten an andere Konsumenten weitergeben können.

Dass Konsumenten ihre Kaufentscheidungen an den genannten Produkturteilen ausrichten, setzt voraus, dass sie die Verbraucherorganisationen und ihre Bewertungen kennen und ihnen vertrauen. Dass Utopia monatlich 80.000 Plattform-Nutzer zählt, die VI 8000 individuelle und 176 kollektive Mitglieder umfasst und auch die Zeitschriften der STW massenhaft bezogen werden (Schrader 2008, S. 161), kann als deutliches Indiz dafür gewertet werden, dass Konsumenten die Produkturteile der Verbraucherorganisationen durchaus wahrnehmen. Und empirische Studien zeigen, dass Konsumenten die Verbraucherorganisationen kennen sowie ihnen insgesamt ein hohes Maß an Vertrauen entgegenbringen. ${ }^{5}$ Durch ihre Produktbewertungen tragen sie dazu bei, die Unsicherheit von Konsumenten über sozial-ökologische Qualitäten abzubauen und ihnen die Unterscheidung zwischen nachhaltigen und konventionellen Gütern zu erleichtern (Fricke und Schrader 2014). Die zunehmende Berücksichtigung von nachhaltigen Produkten in den Publikationsorganen von Verbraucherorganisationen und ihre Beurteilung stellen zugleich eine Art Meta-Klassifizierung dar, denn im Gegensatz zu Labels wird nicht nur bewertet, ob Angebote bestimmte sozial-ökologische Kriterien erfüllen, sondern es werden auch Unterschiede zwischen mehr oder weniger nachhaltigen Produkten deutlich gemacht.

Die dritte hier in den Blick zu nehmende Strategie von Verbraucherorganisationen ist die Beratung von Firmen hinsichtlich ihrer Nachhaltigkeitsstrategie. Ein solcher Unternehmensdialog wird insbesondere von Utopia und der VI durchgeführt und durch kostenpflichtige Unternehmensberatungen (Utopia, VI), Beiratstätigkeiten (VI) und Stakeholderprozesse (Utopia, VI) umgesetzt. Diese Strategie zielt darauf ab, Konsumentenerwartungen an Firmen direkt oder indirekt weiterzuleiten. Eine direkte Kommunikation zwischen Anbietern und Konsumenten wird von Utopia über eine Social-Media-Plattform auf ihrer Internetseite ermöglicht. Im Rahmen von kostenpflichtigen Unternehmensprofilen berichten Firmen auf der Utopia-Website über ihre Nachhaltigkeitsstrategie und über ihre Produkte. Derzeit nehmen 32 Firmen

\footnotetext{
5 So geben Konsumenten in einer aktuellen Bevölkerungsumfrage an, dass ihnen die STW und die Vz zu $95 \%$ bekannt sind. Und auch die (wesentlich kleinere) VI kennen immerhin noch $38 \%$. Auf die Frage, wer die „Interessen von Konsumenten schützen“ kann und am „,meisten dafür tut“, nennen $74 \%$ die Verbraucherzentralen, $64 \%$ die STW und $38 \%$ andere Verbraucherorganisationen wie Foodwatch und die VI (FES 2016, S. 17).
} 
diese Möglichkeit wahr, darunter auch einige Großunternehmen. Die Unternehmensprofile sind wie ein Blog aufgebaut. Auf einer „Pinnwand“ stellen Anbieter nachhaltige Produkte und ihre Nachhaltigkeitsstrategie vor. Der direkte Dialog zu den Konsumenten wird über eine Kommentarfunktion hergestellt, über die UtopiaNutzer ihre Meinung direkt an Firmen kommunizieren können und diese hierauf antworten. In ihren Beratungs- und Beiratstätigkeiten sowie in Stakeholderprozessen nehmen Utopia und die VI hingegen eine Stellvertreterrolle für die Konsumenten ein. Diese Praxis wird im Folgenden anhand von Stakeholderprozessen exemplarisch verdeutlicht.

Stakeholderprozesse bezeichnen Dialoge zwischen Firmen und gesellschaftlichen Anspruchsgruppen ihres Handelns wie Gewerkschaften, NGOs oder Verbraucherorganisationen (Nessel 2015). Die VI führt solche Dialoge u. a. im Projekt „Nachhaltig einkaufen“ durch. Ausgangspunkt dieses Projektes waren zwei von den Initiatoren festgestellte Aspekte, die exemplarisch auch das „Koordinationsproblem“ nachhaltiger Akteure verdeutlichen: Auf der einen Seite zeigte sich, „dass Verbraucher von den verschiedenen Kommunikationsangeboten der Unternehmen verunsichert oder durch diese nicht erreicht werden. [...] Auf der anderen Seite sind viele Unternehmen unsicher, wie sie ihr CSR-Engagement verbrauchergerecht und glaubwürdig an ihre Kunden kommunizieren können. "6 Um dieses Koordinationsproblem anzugehen, wurden zunächst Konsumentenbefragungen durchgeführt, die Anbietern Verbrauchererwartungen verdeutlichen sollten. Auf dieser Grundlage wurde dann ein „Orientierungsrahmen für eine verbrauchergerechte CSR-Kommunikation“ erarbeitet, der „Vertretern aus Gesellschaft [NGOs], Politik [Umweltbundesamt] und Wirtschaft zur Diskussion gestellt“ wurde. Schließlich wurden „Leitlinien für eine zielgruppenspezifische, handlungsorientierte und verbrauchergerechte CSRKommunikation erarbeitet“. Ein ähnlicher Stakeholderprozess wird von der UtopiaStiftung mit dem „Changemaker-Projekt“" umgesetzt, das u.a. von der Deutschen Bundesstiftung Umwelt gefördert wurde. Anbieter verpflichten sich im Rahmen dieses Projekts zur Umsetzung von Nachhaltigkeitsstrategien, z. B. zur „Integration von nachhaltigen Prinzipien in Managementprozesse und die Wertschöpfungskette“, zur „Reduktion von Schadstoffemissionen“ oder zum „Übertreffen von Sozialstandards einer Branche“. ${ }^{7}$ Diese Selbstverpflichtungen werden jährlich von der UtopiaStiftung und einem Beirat aus Nachhaltigkeitsexperten überprüft und mit den beteiligten Firmen diskutiert. Firmen, die diese Ziele erfüllen, werden dann mit dem Titel „Changemaker“ ausgezeichnet.

Verbraucherorganisationen nehmen in Unternehmensdialogen eine Vermittlerposition zwischen den Erwartungen von Konsumenten hinsichtlich der sozial-ökologischen Eigenschaften von Produkten und der ,glaubhaften“ Kommunikation dieser Aspekte seitens der Anbieter ein. Sie kanalisieren sozial-ökologische Verbrauchererwartungen an Firmen entweder mittelbar, indem sie durch Beiratstätigkeiten, Stakeholderprozesse oder kostenpflichtige Beratungen stellvertretend für die Konsumen-

\footnotetext{
6 Vgl. für dieses und die nachfolgenden Zitate http://www.nachhaltig-einkaufen.de/unternehmenskommunikation/unternehmens-kommunikation. Zugegriffen: Okt. 2016.

7 Vgl. http://www.utopia-changemaker.de/wp-content/uploads/2013/04/Changemakermanifest_ blanko2013.pdf. Zugegriffen: Okt. 2016.
} 
ten sprechen, oder unmittelbar, indem sie Angebote bereitstellen, die einen direkten Dialog zwischen Firmen und Konsumenten ermöglichen. Folgt man der vielbeachteten Argumentation Albert O. Hirschmans (1970), dann liefern solche qualitativen Informationen (,voice“) Firmen wichtige Ressourcen zur Ausrichtung ihrer Produkte an den Erwartungen von Konsumenten. Dass die VI und Utopia nicht nur selbst Unternehmensdialoge initiieren, sondern Firmen sich von ihnen auch kostenpflichtig beraten lassen, verdeutlicht, dass Anbieter durch die Kooperation mit Verbraucherorganisationen genauere Kenntnisse über Konsumentenerwartungen einholen und versuchen, ihre Strategien und Produkte hieran auszurichten. Unternehmensdialoge tragen so dazu bei, die Koordination zwischen den Marktseiten zu begünstigen, indem sie Firmen durch direkte oder indirekte Rückmeldungen der Konsumentenseite konkrete Hinweise über die Wahrnehmung ihrer Produktstrategien vermitteln. Zugleich zeigen sie den Firmen, dass Konsumenten durchaus eine Zahlungsbereitschaft für nachhaltige Produkte haben. Die Dialoge können Unternehmen so dazu motivieren, verstärkt sozial-ökologische Produkte anzubieten.

\section{Verbraucherpolitik und Nachhaltigkeit}

In den vorangegangenen Ausführungen wurde am Beispiel von Verbraucherorganisationen die organisationale Dimension nachhaltiger Verhaltensweisen in den Blick genommen. In diesem Abschnitt des Beitrags werden politische Maßnahmen zur Förderung des nachhaltigen Konsums verdeutlicht.

Bis in die 1980er-Jahre richtete sich die staatliche Verbraucherpolitik am Leitbild von „Wettbewerb und Information“ aus. Grundlage dieses Leitbildes war die Vorstellung von Konsumenten und Firmen als ökonomisch rationale Akteure. Hinsichtlich der Konsumenten wurde davon ausgegangen, dass sie bei Vorliegen möglichst vollständiger Informationen ihre Interessen im Sinne der Konsumentensouveränität bestmöglich verfolgen könnten und so zu ökonomisch effizienten Märkten beitragen. Dementsprechend konzentrierten sich die politischen Maßnahmen vor allem darauf, das Informationsniveau der Konsumenten zu erhöhen (z. B. durch das „,Gesetz zur Regelung der Allgemeinen Geschäftsbedingungen“ und das „Gesetz gegen unlauteren Wettbewerb“). ${ }^{8}$ Insbesondere seit der Rio-Konferenz 1992 und der BSEKrise 2001 haben sich jedoch das verbraucherpolitische Leitbild und die ihm zugrundeliegende Akteurskonzeption weiter ausdifferenziert. So stimmen der wissenschaftliche Beirat des BMELV (Schrader et al. 2013), die Bundesregierung (2012) sowie wissenschaftliche Beobachter (Belz und Billharz 2007; Müller 2001; Reisch 2005) darin überein, dass die Rolle von Marktteilnehmern auch sozial-ökologisch definiert werden müsse und sich politische Maßnahmen am Leitbild des mündigen und verantwortungsvollen Verbrauchers und Anbieters ausrichten sollen. Dieses Leitbild wurde dann durch die nationale Nachhaltigkeitsstrategie 2002 und den Aktionsplan Verbraucherschutz 2003 institutionalisiert. So heißt es in Letzterem: „Die Bundesregierung hat sich das Ziel gesetzt, Verbraucher und Anbieter gleichermaßen

\footnotetext{
${ }^{8}$ Zur Begründung verbraucherpolitischer Leitbilder und zu ihrer Wirkung auf politische Maßnahmen vgl. Kuhlmann 1990, S. 27 ff.; Mitropoulos 1997, S. 18 ff.; Nessel 2016, S. 76 ff.
} 
$\mathrm{zu}$ [Nachhaltigkeit zu] aktivieren und strebt ein hohes Maß an Transparenz hinsichtlich der Herstellungsprozesse an." Darüber hinaus zielt die Bundesregierung darauf ab, „Fragen in Zusammenhang mit einer nachhaltigen Entwicklung stärker in das Bewusstsein der Öffentlichkeit zu bringen“" (Bundesregierung 2003, S. 20). Diese Ziele sind bis heute politisch bedeutsam und haben auch Eingang in den aktuellen Koalitionsvertrag gefunden, wonach „Nachhaltigkeit für die neue Bundesregierung [...] grundlegendes Ziel und Maßstab des Regierungshandelns“" sei (Merkel 2014).

Im Rahmen einer neuen, um Nachhaltigkeitsziele erweiterten Verbraucherpolitik (Müller 2001; Reisch 2005) unternehmen politische Akteure seitdem zahlreiche Anstrengungen, um nachhaltige Verhaltensweisen zu befördern. Im Jahr 2001 hat die Bundesregierung ein einheitliches Siegel für ökologische Produkte eingeführt: das (deutsche) Bio-Siegel. Damit wurde eine rechtliche Regulierung geschaffen, die Anbieter darauf verpflichtet, einheitliche Mindeststandards bei der Produktion biologischer Lebensmittel einzuhalten. Im März 2015 nutzten 4486 Unternehmen das Siegel auf 70.393 Produkten. ${ }^{9}$ Auch das älteste bundesdeutsche Label für umweltverträgliche Produkte, der „Blaue Engel“, wird vom Bund gegenwärtig verstärkt gefördert, um Konsumenten die Auswahl ökologischer Produkte zu erleichtern. ${ }^{10} \mathrm{Um}$ staatliche Labels und das Konzept der Nachhaltigkeit in der Bevölkerung bekannt zu machen, führen staatliche Stellen darüber hinaus regelmäßig Aufklärungs- und Informationskampagnen durch. Solche Kampagnen beinhalten Anzeigen in Zeitungen, auf Werbeplakaten sowie Ratgeber zu nachhaltigem Konsum (z. B. der „Nachhaltige Warenkorb“).

Der Staat tritt immer häufiger auch selbst als Anbieter und Nachfrager sozialökologischer Produkte auf. Seit 2010 sind öffentliche Einrichtungen dazu angehalten, bei der Beschaffung nicht nur Preis-, sondern auch Nachhaltigkeitsaspekte zu berücksichtigen (Bundesregierung 2012, S. 43 ff.). Solche Vorgaben sind einerseits wichtig, um zu verdeutlichen, dass die Politik nicht nur von den Konsumenten und Anbietern ein nachhaltiges Verhalten einfordert, sondern auch selbst Maßnahmen zu dessen Umsetzung unternimmt (Schrader et al. 2013, S. 10). Andererseits tragen solche Vorgaben dazu bei, Firmen durch ökonomische Anreize zur Herstellung nachhaltiger Produkte zu motivieren. In diesem Sinne beeinflusst der Staat ,auch über seine Beschaffungspolitik, welche Angebote am Markt Erfolg haben und damit zukünftig verstärkt angeboten werden können und welche nicht. Von daher ist der Eingang sozialer und ökologischer Kriterien in staatliche Beschaffungsrichtlinien ein wesentliches Instrument zur Förderung nachhaltiger Angebote“. Darüber hinaus ,agiert der Staat auch als Anbieter bzw. kann das Angebot direkt beeinflussen. Hier ist beispielsweise das Nahrungsmittelangebot in öffentlichen Einrichtungen angesprochen." (ebd.).

Die Erweiterung des verbraucherpolitischen Leitbilds um Nachhaltigkeitsaspekte wirkt sich auch auf die Verbraucherbildung aus. So wurde das Projekt „Schulstart für den Blauen Engel - $100 \%$ für unsere Umwelt“ etabliert, das Eltern und Schüler

\footnotetext{
9 Vgl. http://www.oekolandbau.de/bio-siegel/. Zugegriffen: Okt. 2015.

$10 \mathrm{Vgl}$. https://www.bundesregierung.de/Content/DE/Artikel/2016/10/2016-10-25-erster-aktionstagblauer-engel.html. Zugegriffen: Okt. 2015.
} 
„für einen nachhaltigen Konsum sensibilisieren“ soll. Hierzu wurden Unterrichtsmaterialien verteilt, damit „Schüler das Umweltzeichen kennen, erfahren, wie dadurch die Umwelt geschützt wird, [...] [und] was sie mit dem Blauen Engel für die Umwelt tun können“ (Bundesregierung 2013, S. 27). Durch das BMELV wurde zudem das Projekt „CSR und Verbraucherinformation“ umgesetzt, das ebenfalls darauf abzielt, „Schülerinnen und Schüler unterschiedlicher Schulstufen und Schultypen“ für Nachhaltigkeitsaspekte zu ,sensibilisieren“ (ebd.). Und zusammen mit dem vzbv hat das BMELV die Initiative „Verbraucherbildung - Konsumkompetenz stärken“ ins Leben gerufen, die eine Verankerung von Verbraucherbildung in Schulen zum Ziel hat.

Bund und Länder fördern seit einiger Zeit verstärkt auch NGOs und Verbraucherorganisationen, um nachhaltige Handlungsabsichten und Einstellungen anzuregen. So wurden mittels öffentlicher Finanzierung in Bayern z. B. Energiestützpunkte aufgebaut, die durch die Vz Bayern verwaltet werden und die Konsumenten für Energieeinsparmaßnamen sensibilisieren sowie zur Umsetzung eines energiesparenden Konsums beraten sollen. Und die Deutsche Stiftung Verbraucherschutz wurde durch das BMELV mit zehn Millionen Euro Stiftungskapital eingerichtet. Die Wirkung von Verbraucherorganisationen und NGOs auf Nachhaltigkeit anerkennend, folgert die Bundesregierung (2003, S. 18): Sie ,,beteiligen sich [...] aktiv und öffentlichkeitswirksam an der Diskussion über die Entwicklung und Umsetzung des Leitbildes einer nachhaltigen Entwicklung [...] und bieten entsprechende Bildungsmaßnahmen an. [...] Zudem erreichen Nichtregierungsorganisationen u. a. durch ihre Initiativen vor Ort oft Zielgruppen, die Bildungsinstitutionen weniger zugänglich sind.“ Zur Umsetzung von Nachhaltigkeit wurden bis zum Jahr 2015 ca. 8600 Projekte von NGOs und Verbraucherorganisationen mit rund 1,5 Mrd. Euro gefördert. ${ }^{11}$

Schließlich ist darauf hinzuweisen, dass Bund und Länder in jüngster Zeit verstärkt versuchen, ihre Nachhaltigkeitsstrategie durch Beratungsgremien wissenschaftlich zu fundieren. So wurden auf Bundesebene der Rat für nachhaltige Entwicklung und der parlamentarische Beirat für Nachhaltigkeit eingerichtet. Diese Gremien sollen Handlungsempfehlungen für Bundesministerien und -behörden erarbeiten, um nachhaltigen Konsum zu fördern und umzusetzen. Neuerdings soll der Sachverständigenrat für Verbraucherfragen ,evidenzbasierte“ Hinweise für die Verbraucherpolitik liefern. Ähnliche Gremien finden sich in den Bundesländern. ${ }^{12}$ Seitdem die Politik Nachhaltigkeit als verbraucherpolitisches Ziel aufgegriffen hat, setzten auch immer mehr Ausschreibungen für institutionelle Förderungen einen Bezug zum Thema voraus, wodurch die wissenschaftliche Forschung zu nachhaltigem Konsum weiter angeregt wird (BBF 2008).

Die dargestellten Beispiele sollten in ihrer Zusammenschau exemplarisch die Neuausrichtung der deutschen Verbraucherpolitik verdeutlichen, die auf dem Leitbild des (ökonomisch) mündigen und (sozial-ökologisch) verantwortungsvollen Ak-

\footnotetext{
11 Vgl. http://www.bundesregierung.de/Webs/Breg/DE/Themen/Nachhaltigkeitsstrategie/3-nachhaltigeentwicklung-alle-sind-Partner/Verbaende-Stiftungen/_node.html. Zugegriffen: Okt. 2016.

12 Bund und Länder beteiligen sich auch an kommunalen „Agenda 21“-Prozessen und weiteren Initiativen auf lokaler Ebene. Vgl. http://www.bmub.bund.de/themen/strategien-bilanzen-gesetze/nachhaltigeentwicklung/lokale-agenda-21/. Zugegriffen: Okt. 2015.
} 
teurs gründet. Vor dem Hintergrund dieses Leitbildes wurden diverse politische Maßnahmen umgesetzt, die sich auf das Wissen der Konsumenten über Nachhaltigkeit im Allgemeinen (Kampagnen, Bildungsarbeit und Ratgeber) sowie über nachhaltige Produkte im Speziellen (Ratgeber und Labels) beziehen. Darüber hinaus wurden neue Institutionen gegründet (Nachhaltigkeitsbeiräte) oder bestehende Institutionen verändert (öffentliche Beschaffung). Durch diese Maßnahmen nimmt der Staat Einfluss auf die institutionellen und kulturellen Rahmenbedingungen nachhaltigen Konsums und damit auf die Bedingungen, unter denen Konsumenten und Anbieter handeln.

\section{Verbraucherorganisationen und Verbraucherpolitik als Intermediäre nachhaltigen Konsums}

In den vorangegangenen Ausführungen wurde argumentiert, dass die Handlungsabsichten, die Einstellungen, das Wissen sowie die Koordination von Konsumenten und Firmen wichtige Faktoren nachhaltigen Konsums sind, die wesentlich durch das soziale Umfeld der Akteure beeinflusst werden. Diese soziale Einbettung des nachhaltigen Konsums wurde am Beispiel von Verbraucherorganisationen und der Verbraucherpolitik dann genauer verdeutlicht. Abschließend wird nun gezeigt, dass verbraucherpolitische Akteure Intermediäre der Nachhaltigkeit sind, die zentrale Voraussetzungen für einen sozial-ökologischen Konsum schaffen.

Motivationen und Einstellungen wurden in diesem Beitrag als erster zentraler Faktor nachhaltigen Konsums sichtbar gemacht. Zudem wurde verdeutlicht, dass ihre Entstehung ein allgemeines Wissen über Nachhaltigkeit und ein spezifisches Wissen über nachhaltige Produkteigenschaften voraussetzt. Dieses Nachhaltigkeitswissen stellt den zweiten zentralen Faktor nachhaltigen Konsums dar, denn es legt den Grundstein dafür, dass Konsumenten nachhaltige Angebote als solche wahrnehmen und ein Problembewusstsein für die Auswirkungen ihres Konsums auf die Umwelt und auf Andere im Sinne der Nachhaltigkeit (sozial-ökologische Einstellungen und Absichten) oder auf das eigene Selbst (zweckrationale Einstellungen und Absichten) entwickeln. Ein solches Wissen kann allerdings schwerlich allein auf individueller Ebene entstehen. Vielmehr wird es u. a. durch wissenschaftliche Studien (Steg 2015) und soziale Bewegungen (Micheletti und Stolle 2007) generiert sowie über die Medien (Thøgersen 2005) und über private Haushalte (Matthies und Wallis 2015) vermittelt. In diesem Beitrag wurde gezeigt, dass auch verbraucherpolitische Akteure Nachhaltigkeitswissen sowohl generieren als auch verbreiten. Allen deutschen verbraucherpolitischen Akteuren ist gemeinsam, dass sie Ratgeber, Broschüren und diverse andere Publikationen herausgeben sowie Webseiten betreiben, in bzw. auf denen nachhaltige Verhaltensweisen konkretisiert sowie Effekte des Konsums auf Umwelt und Gesellschaft problematisiert werden. Eine ähnliche Vorgehensweise findet sich in Projekten zur schulischen und außerschulischen Nachhaltigkeitsbildung, die von den Vz, dem vzbv sowie von den Ministerien der Bundes- und Landesebene umgesetzt werden, sowie in Projekten zur Erwachsenenbildung der Vz und der VI. Insbesondere staatliche Stellen führen darüber hinaus Aufklärungskampagnen zum Thema Nachhaltigkeit durch. 
Diese Praktiken zeigen einerseits mögliche negative Auswirkungen von Konsum und Produktion auf Umwelt und Gesellschaft auf. Ein solches „framing“ von Nachhaltigkeit als ökologisches und soziales Problem ist eine zentrale Voraussetzung, um ein Verständnis für den Beitrag einzelner (Konsum-)Handlungen für eine nachhaltige Einwicklung zu schaffen und um Konsumenten - sowie auch Anbieter - zur Übernahme von Verantwortung hierfür zu motivieren (Micheletti und Stolle 2007; Thøgersen 2005, S. 159 f.). Indem verbraucherpolitische Akteure öffentlich abrufbare Publikationen herausgeben, Kampagnen weithin sichtbar sind und auch die Massenmedien über ihre Aktivitäten berichten, wird Nachhaltigkeitswissen nicht nur in spezifischen sozialen Milieus (z. B. den sogenannten LOHAS, den Konsumenten mit einem Lifestyle of Health and Sustainability) verbreitet, sondern auch in einer breiten Öffentlichkeit. Andererseits konkretisieren diese Praktiken das Konzept der Nachhaltigkeit, indem auf die Möglichkeit zum Kauf nachhaltiger Produkte hingewiesen und ihre Kennzeichnung verdeutlicht wird. Solche Konkretisierungen sind bedeutsam, um ein Wissen über Klassifizierungen von Produkten und ihren Nachhaltigkeitscharakter zu generieren, durch das diese Bewertungen kaufrelevant werden können (Thøgersen 2005, S. 152). Zusammen mit sozialen Bewegungsorganisationen (Balsiger 2010; Micheletti und Stolle 2007) sowie wissenschaftlichen Akteuren (Steg 2015) schaffen verbraucherpolitische Akteure damit zentrale Voraussetzungen für die Entstehung sozial-ökologischer Motivationen und Einstellungen. Wie diese Betrachtungen zeigen, sind diese beiden zentralen Faktoren des nachhaltigen Konsums - die Handlungsbereitschaft und das Nachhaltigkeitswissen der Konsumenten - nicht allein auf sozialstrukturelle Eigenschaften zurückzuführen oder einfach als gegeben vorauszusetzen. Vielmehr werden sie durch Organisationen sozial vermittelt und aktiv beeinflusst. Indem verbraucherpolitische Akteure den Nachhaltigkeitscharakter von Produkteigenschaften (Anbaumethode, Herstellungsprozess etc.) verdeutlichen und z. B. Bio-Produkte als ,gesünder“, „sozialverträglicher“ und „umweltschonender“ rahmen, beeinflussen sie zugleich die zweckrationalen Handlungsabsichten und Einstellungen der Konsumenten. Ein Wissen bzw. ein Glaube an die ,gesundheitsfördernde“" Wirkung von Bio-Produkten kann nicht auf individueller Ebene allein entstehen, sondern wird in entscheidendem Maße durch die soziale Umwelt von Akteuren mitkonstituiert (Magnusson et al. 2003). Dementsprechend sind ,consumer information and education [...] not less relevant in empowering consumers to look out for their sustainability interests than for their health [...] related interests“ (Thøgersen 2005, S. 155).

Die verbraucherpolitischen Akteure verankern durch die genannten Praktiken zugleich die Vorstellung des mündigen und verantwortungsvollen Konsumenten und Anbieters als ein gesellschaftliches Leitbild. Da Leitbilder Anforderungen an das Handeln von Akteuren und „Utopien“ über wünschenswerte Verhaltensweisen formulieren, beeinflussen sie die kulturelle Einbettung von Konsumenten und Anbietern - und können hierdurch handlungsrelevant werden (Kern 2014; Kuhlmann 1990, S. 19 ff.). Wie am Beispiel der deutschen Verbraucherpolitik gezeigt wurde, führte die Erweiterung des verbraucherpolitischen Leitbildes um Nachhaltigkeitsaspekte dazu, dass neue Beratungsgremien auf Bundes- und Landesebene gegründet und wissenschaftliche Studien zur Nachhaltigkeit sowie Aktivitäten von Verbraucherorganisationen verstärkt finanziell gefördert wurden. Hierdurch wird die Produktion 
weiteren Nachhaltigkeitswissens angeregt und dieses verbreitet. Das Leitbild des verantwortungsvollen Akteurs anwendend, setzt auch die staatliche Politik immer mehr auf eine nachhaltige öffentliche Beschaffung (Schrader et al. 2013, S. 10). Durch diese veränderte institutionelle Rahmenbedingung werden auch Firmen verstärkt zum Angebot nachhaltiger Güter motiviert.

Einstellungen und Motivationen sind ein wichtiger, aber kein hinreichender Faktor nachhaltigen Konsums. Wie in diesem Beitrag gezeigt wurde, setzt die Verwirklichung nachhaltigen Konsums weiter voraus, dass Konsumenten sozial-ökologische Produkte erkennen und Unsicherheit über ihre Qualität abbauen. Sozial-ökologische Qualitäten von Produkten sind für Konsumenten jedoch selbst kaum nachprüfbar (Fricke und Schrader 2014), und auch Preise geben kaum Auskunft darüber, ob ein Produkt mehr oder weniger nachhaltig ist. Konsumenten sind daher auf externe und vertrauenswürdige Wissensbestände angewiesen, um die Qualität von Angeboten einschätzen zu können. In den vorangegangenen Ausführungen wurde verdeutlicht, dass verbraucherpolitische Akteure durch Produktbewertungen und -klassifikationen anbieterunabhängige und glaubhafte Informationen über Gütereigenschaften bereitstellen. Kennzeichnend für die Bewertungspraxis von Verbraucherorganisationen ist einerseits, dass sie nachhaltige Produkte von konventionellen unterscheiden, indem nur erstere in ihre Publikationen aufgenommen werden. Andererseits machen sie Unterschiede zwischen nachhaltigen Angeboten kenntlich, indem sie diese detailliert beschreiben oder ein „Ranking“ zwischen ihnen vornehmen. Konsumenten erreichen diese Bewertungen vielfach auf indirektem Weg, insbesondere durch öffentlich einsehbare Internetseiten, Mitgliederzeitschriften oder Ratgeber, teilweise aber auch über die Massenmedien, die über ausgewählte Testergebnisse berichten, sowie über die Hersteller, die damit werben. Die staatliche Verbraucherpolitik klassifiziert und bewertet Produkte hingegen durch die Vergabe von Labels wie dem deutschen BioSiegel oder dem Blauen Engel. Eine Besonderheit dieser Praxis ist, dass Hersteller diese Zertifizierungen direkt auf ihren Produkten anbringen und nachhaltige Güter so direkt am Verkaufsort identifizierbar werden. Darüber hinaus werden Hersteller, die mit diesen Labels werben und ihre Angebote von der staatlichen Politik zertifizieren lassen, zur Einhaltung rechtlich verbindlicher Mindeststandards in der Produktion bewogen. Allerdings werden durch diese Zertifizierungen keine Unterschiede zwischen den gelabelten Produkten deutlich.

Durch Produkturteile vermindern verbraucherpolitische Akteure die Unsicherheit über nachhaltige Qualitätseigenschaften. Sie erhöhen so das Produktwissen von Konsumenten und tragen dazu bei, eine wesentliche Barriere nachhaltiger Kaufentscheidungen zu überwinden, nämlich den Informations- und Zeitaufwand bei der Auswahl nachhaltiger Produkte (Tanner und Kast 2003, S. 893). Durch Produkturteile wird zugleich die Übersetzung von Absichten und Einstellungen in nachhaltige Verhaltensweisen begünstigt, da hierdurch für die Konsumenten die kognitiven Anforderungen bei der Suche nach sozial-ökologischen Angeboten reduziert werden (Young et al. 2010, S. 22 ff.). Im Anschluss an Karpik (2010, S. 62 ff.) sind verbraucherpolitische Akteure daher als Instanzen der Urteilsbildung auf nachhaltigen Märkten zu kennzeichnen, die Konsumenten die Auswahl nachhaltiger Produkte ermöglichen und die Koordination der Marktseiten begünstigen. Hinzuweisen ist schließlich darauf, dass positive Produkturteile von Anbietern genutzt werden kön- 
nen, um Konsumenten die sozial-ökologischen Qualitätseigenschaften ihrer Produkte glaubhaft zu bescheinigen (Fricke und Schrader 2014). Firmen werben daher mit „guten“ Testergebnissen (Schrader 2008) oder mit Preisen, mit denen sie für ihr nachhaltiges Engagement ausgezeichnet wurden.

Damit Konsumenten ihre Kaufentscheidungen an Urteilen von Verbraucherorganisationen ausrichten können, müssen sie diese kennen und ihnen vertrauen. Da beides durchaus verbreitet der Fall ist (FES 2016), können Produkturteile von Verbraucherorganisationen somit auch kaufrelevant werden (Umweltbundesamt 2014, S. 48 ff.). Entsprechend ihrer kooperativen Ausrichtung verzichten Utopia und die VI allerdings ebenso wie staatliche Akteure darauf, ,weniger nachhaltige“ Produkte beim Namen zu nennen. Sie folgen damit der Überlegung, dass positive Anreize zur Umsetzung von Nachhaltigkeit (,,buycott“) besser geeignet seien als das „blaming“ von Firmen. Einzig durch die STW werden Nachhaltigkeitsversprechen auch öffentlich kritisiert, da ihre CSR-Tests positiv und negativ beurteilte Produkte abbilden. Wie erste empirische Studien nahelegen, scheinen insbesondere positive (CSR-)Urteile (Schrader 2008) und eine Kenntnis von privaten und staatlichen Labels (Umweltbundesamt 2014, S. 48 ff.) die Verkaufszahlen von sozial-ökologischen Angeboten zu erhöhen. Wie Schrader (2008, S. $161 \mathrm{f}$.) am Beispiel der STW folgert, ,ist dieser direkte Einfluss [von Produkturteilen] [jedoch] nicht der Einzige und vermutlich nicht mal der entscheidende. [...] Allein durch die Tätigkeit der Stiftung und das Risiko eines schlechten Abschneidens der Anbieter besteht für die Unternehmen ein Anreiz, die [sozial-ökologische] Qualität ihrer Produkte zu verbessern. " ${ }^{13}$

Den möglichen Effekten des Nachhaltigkeits- und Produktwissens sind allerdings Grenzen gesetzt. In Bezug auf das Nachhaltigkeitswissen ist darauf hinzuweisen, dass die (Massen-)Medien zwar vielfach über die Aktivitäten verbraucherpolitischer Akteure berichten und so ihre Forderungen und Hinweise zum Einbezug nachhaltiger Verhaltensweisen verbreiten. Allerdings berichten die Medien auch über Nachhaltigkeitsskandale und über die Verfehlungen von Anbietern, insbesondere wenn ihre teils auch von verbraucherpolitischen Akteuren bewerteten Produkte Nachhaltigkeitsstandards nicht einhalten (Thøgersen 2005, S. 155 f.). Solche negativen Medienberichte können dazu führen, dass Konsumenten nicht nur sozial-ökologische Produkteigenschaften anzweifeln, sondern auch den verbraucherpolitischen Angeboten zur Umsetzung von Nachhaltigkeit mit einem größeren Misstrauen begegnen. Hinsichtlich des möglichen Produktwissens ist darauf hinzuweisen, dass CSR-Tests der STW sowie Bewertungen von Utopia und der VI nicht alle Angebote einer Branche einbeziehen. Auch können aufgrund hoher Testkosten oder sich schnell wandelnder Angebote nicht alle Produkte eines Marktbereichs in gleicher Weise getestet werden. Gerade in Märkten mit hoher Innovationsdynamik, wie z. B. Telekommunikation oder Finanzangeboten, ist es für Verbraucherorganisationen nahezu unmöglich, aktuelle Produkturteile zu formulieren. Auch legen erste Studien nahe, dass ihre Bewertungen nicht alle Konsumenten erreichen, sondern vor allem die besser gebildeten und ökonomisch besser situierten Schichten (Stolle und Micheletti 2013, S. 60 ff.). Darüber hinaus müssen Konsumenten oft aus einer Vielzahl von Produktwertungen auswählen. Diese Überlegung verweist darauf, das verbrau-

${ }^{13} \mathrm{Zu}$ den indirekten Wirkungen von Produkturteilen vgl. auch Nessel 2016, S. 219 f. 
cherpolitische Akteure das Unsicherheitsproblem von Konsumenten über sozialökologische Qualitäten gewissermaßen auf eine andere Ebene verlagern, indem sie eine Vielzahl neuer Informationen produzieren, die die Konsumenten bei ihren Käufen berücksichtigen könnten. In weiteren Studien ist daher noch genauer zu klären, welche Bewertungen welche Konsumentengruppen erreichen, in welchen Marktbereichen sie mehr oder weniger kaufrelevant werden und wie Konsumenten mit der Vielfalt an Produkturteilen oder mit kritischen Medienberichten umgehen.

Wie in diesem Beitrag herausgearbeitet wurde, bedarf es zur Realisierung nachhaltiger Konsummuster schließlich einer regelmäßigen und kooperativen Zusammenführung von Konsumenten und Anbietern. Die Voraussetzungen einer solchen Koordination wurden in der Literatur bisher jedoch nur unzureichend analysiert. Zwar wurde argumentiert, dass soziale und ökologische Labels das Koordinationsproblem abbauen oder dass eine ,glaubhafte Kommunikation“ der Anbieter und ihre Zusammenarbeit mit Verbraucherorganisationen Konsumenten- und Firmenerwartungen in Einklang bringen können (Fricke und Schrader 2014). Viele Studien vermitteln aber den Eindruck, dass es bei transparenten Produktmerkmalen und ihrer glaubhaften Vermittlung nahezu reibungslos zu einem einvernehmlichen Zusammenspiel der Marktseiten käme. Hierbei wird übersehen, dass eine dauerhafte Koordination von Marktakteuren daran gebunden ist, dass sie die Erwartungen der jeweils Anderen angemessen einschätzen und Unsicherheit darüber abbauen müssen (Beckert 2007). Um ihre Erwartungen zu stabilisieren, können Konsumenten, wie gezeigt, auf Produkturteile verbraucherpolitischer Akteure zurückgreifen. Anbieter können hingegen Marktforschung betreiben oder die Kauf- bzw. Nichtkaufentscheidungen (,exit/buy“-Entscheidungen) der Konsumenten hinsichtlich ihrer Produkte ex post auswerten. Eine andere Strategie von Firmen wurde in diesem Beitrag am Beispiel der Unternehmensdialoge von Utopia und der VI verdeutlicht. Durch die Zusammenarbeit mit beiden Organisationen versuchen Firmen, Konsumentenerwartungen abzuschätzen und ihre Produktstrategien genauer an ihnen auszurichten. Hierzu lassen sie sich kostenpflichtig beraten, führen Stakeholderprozesse durch und integrieren z. B. die VI in Unternehmensbeiräte. Wie Hirschman (1970) verdeutlicht hat, gibt eine Auswertung von ,exit/buy“-Entscheidungen den Anbietern kaum konkrete Hinweise darauf, warum Konsumenten Güter kaufen oder warum nicht. Dementsprechend hat Hirschman die qualitative Rückmeldung von Konsumenten (,,voice“) als zentrales Instrument zur Optimierung von Produktstrategien und zur Koordination der Marktseiten bezeichnet. Indem Utopia und die VI über Unternehmensdialoge die Erwartungen der Konsumenten für Firmen sichtbar machen, nehmen sie eine wichtige Vermittlerposition zwischen den beiden Marktseiten ein. Durch Preise für nachhaltiges Engagement, positive Produkturteile oder durch Labels können Firmen Konsumenten die sozial-ökologische Qualität ihrer Produkte glaubhaft(er) bescheinigen. Da verbraucherpolitische Akteure die Nachhaltigkeitsstrategien von Anbietern beobachten und öffentlich abbilden, entsteht für die Firmen darüber hinaus ein öffentlicher Druck, ihre Produktversprechen auch einzuhalten, was sie wiederum zu einer nachhaltigen Produktion motiviert.

Deutsche Verbraucherorganisationen und die staatliche Verbraucherpolitik wurden in diesem Beitrag als ein zentraler Bestandteil des institutionellen, kulturellen und organisationalen Umfeldes von Konsumenten und Anbietern kenntlich gemacht. 
Als Intermediäre nachhaltigen Konsums tragen sie zur Entstehung nachhaltigkeitsrelevanter Motivationen und Einstellungen, zum Wissen über sozial-ökologische Produkte sowie zur Koordination von Konsumenten und Anbietern bei. Nachhaltiger Konsum ist daher nicht allein auf individuelle Faktoren wie Bildung, Geschlecht, Einkommen oder Präferenzen zurückzuführen, sondern muss als eine organisational vermittelte soziale Praxis verstanden werden.

Danksagung Ich danke Frithjof Nungesser, Sandra Pfister und Henri Band für wichtige Hinweise zu diesem Beitrag.

Open Access Dieser Artikel wird unter der Creative Commons Namensnennung 4.0 International Lizenz (http://creativecommons.org/licenses/by/4.0/deed.de) veröffentlicht, welche die Nutzung, Vervielfältigung, Bearbeitung, Verbreitung und Wiedergabe in jeglichem Medium und Format erlaubt, sofern Sie den/die ursprünglichen Autor(en) und die Quelle ordnungsgemäß nennen, einen Link zur Creative Commons Lizenz beifügen und angeben, ob Änderungen vorgenommen wurden.

Open access funding provided by University of Graz.

\section{Literatur}

Balsiger, P. (2010). Making political consumers. The tactical action repertoire of a campaign for clean clothes. Social Movement Studies, 9, 311-329.

Baumann, S., Engman, A., \& Johnston, J. (2015). Political consumption, conventional politics, and high cultural capital. International Journal of Consumer Studies, 39, 413-421.

Beckert, J. (2007). Die soziale Ordnung von Märkten. In J. Beckert, R. Diaz-Bone \& H. Ganßmann (Hrsg.), Märkte als soziale Strukturen (S. 43-62). Frankfurt a. M.: Campus.

Belz, F., \& Bilharz, M. (2007). Nachhaltiger Konsum, geteilte Verantwortung und Verbraucherpolitik. In F.-M. Belz, G. Karg \& D. Witt (Hrsg.), Nachhaltiger Konsum und Verbraucherpolitik im 21. Jahrhundert (S. 21-52). Marburg: Metropolis.

Bluhm, K. (2008). Corporate Social Responsibility. Zur Moralisierung von Unternehmen aus soziologischer Perspektive. In A. Maurer \& U. Schimank (Hrsg.), Die Gesellschaft der Unternehmen Die Unternehmen der Gesellschaft. Gesellschaftstheoretische Zugänge zum Wirtschaftsgeschehen (S. 144-162). Wiesbaden: VS.

Bourdieu, P. (2005). The social structures of the economy. Cambridge: Polity Press.

Bundesministerium für Bildung und Forschung (BBF) (2008). Vom Wissen zum Handeln - Neue Wege zum nachhaltigen Konsum. Berlin: Bundesministerium für Bildung und Forschung.

Bundesregierung (2003). Bericht der Bundesregierung - Aktionsplan Verbraucherschutz. Berlin: Deutscher Bundestag.

Bundesregierung (2012). Verbraucherpolitischer Bericht der Bundesregierung 2012. Berlin: Deutscher Bundestag.

Bundesregierung (2013). Bericht der Bundesregierung zur Bildung für eine nachhaltige Entwicklung. http://dip21.bundestag.de/dip21/btd/17/143/1714325.pdf. Zugegriffen: Okt. 2016.

Fricke, V., \& Schrader, U. (2014). Unternehmenskommunikation zur Förderung des nachhaltigen Konsums. In H. Meffert, P. Kenning \& M. Kirchgeorg (Hrsg.), Sustainable Marketing Management. Grundlagen und Cases (S. 205-226). Wiesbaden: Springer Gabler.

Friedrich-Ebert-Stiftung (FES) (2016). Verbraucherschutz und Verbraucherpolitik im Urteil der Bevölkerung. Eine Repräsentativbefragung der Bevölkerung ab 16 Jahre. Bonn: Friedrich-Ebert-Stiftung.

Heidbrink, L., \& Schmidt, I. (2011). Das Prinzip der Konsumentenverantwortung. Grundlagen, Bedingungen und Umsetzungen verantwortlichen Konsums. In L. Heidbrink, I. Schmidt \& B. Ahaus (Hrsg.), Die Verantwortung des Konsumenten. Über das Verhältnis von Markt, Moral und Konsum (S. 25-56). Frankfurt a. M.: Campus.

Hirschman, A. (1970). Exit, voice, and loyalty. Responses to decline in firms, organizations, and states. Cambridge: Harvard University Press.

Joshi, Y., \& Rahman, Z. (2015). Factors affecting green purchase behaviour and future research directions. International Strategic Management Review, 3, 128-143.

Karpik, L. (2010). Mehr Wert. Die Ökonomie des Einzigartigen. Frankfurt a. M.: Campus. 
Kern, T. (2014). Die Umweltbewegung und der Wandel der institutionellen Logik auf dem Strommarkt. Zeitschrift für Soziologie, 43, 322-340.

Koos, S. (2011). Varieties of environmental labelling, market structures, and sustainable consumption across Europe. A comparative analysis of organizational and market supply determinants of environmental-labelled goods. Journal of Consumer Policy, 34, 127-151.

Kuhlmann, E. (1990). Verbraucherpolitik. Grundzüge ihrer Theorie und Praxis. München: Vahlen.

Lorenz, S. (2006). Biolebensmittel und die „Politik mit dem Einkaufswagen“. In J. Lamla \& S. Neckel (Hrsg.), Politisierter Konsum - konsumierte Politik (S. 91-112). Wiesbaden: VS.

Magnusson, M. K., Arvola, A., Hursti, U. K. K., Åberg, L., \& Sjödén, P. O. (2003). Choice of organic foods is related to perceived consequences for human health and to environmentally friendly behaviour. Appetite, 40, 109-117.

Matthies, E., \& Wallis, H. (2015). Family socialization and sustainable consumption. In L. A. Reisch \& J. Thøgersen (Hrsg.), Handbook of research on sustainable consumption (S. 268-284). Cheltenham: Edward Elgar.

Merkel, A. (2014). Rede von Bundeskanzlerin Merkel auf der 14. Jahreskonferenz des Rates für Nachhaltige Entwicklung. http://www.bundesregierung.de/Content/DE/Rede/2014/06/2014-06-02-merkelnachhaltige-entwicklung.html?nn=549374. Zugegriffen: Okt. 2015.

Micheletti, M., \& Stolle, D. (2007). Mobilizing consumers to take responsibility for global social justice. Annals of the American Academy of Political and Social Science, 611, 157-175.

Micheletti, M., \& Stolle, D. (2012). Sustainable citizenship and the new politics of consumption. Annals of the American Academy of Political and Social Science, 644, 88-120.

Mitropoulos, S. (1997). Verbraucherpolitik in der Marktwirtschaft. Konzeptionen und internationale Erfahrungen. Berlin: Duncker \& Humblot.

Müller, E. (2001). Grundlinien einer modernen Verbraucherpolitik. Aus Politik und Zeitgeschichte, (24), 6-15.

Münch, R. (2008). Jenseits der Sozialpartnerschaft. Die Konstruktion der sozialen Verantwortung von Unternehmen in der Weltgesellschaft. In A. Maurer \& U. Schimank (Hrsg.), Die Gesellschaft der Unternehmen - Die Unternehmen der Gesellschaft (S. 163-190). Wiesbaden: VS.

Nessel, S. (2015). Verbraucherorganisationen als Resilienz- und Vulnerabilitätsfaktor von Markterwartungen. In M. Endreß \& A. Maurer (Hrsg.), Resilienz im Sozialen. Theoretische und empirische Analysen (S. 153-180). Wiesbaden: Springer VS.

Nessel, S. (2016). Verbraucherorganisationen und Märkte. Eine wirtschaftssoziologische Untersuchung. Wiesbaden: Springer VS.

Reisch, L. A. (2003). Strategische Grundsätze und Leitbilder einer neuen Verbraucherpolitik. Diskussionspapier des Wissenschaftlichen Beirats für Verbraucher- und Ernährungspolitik beim BMVEL. Stuttgart: BMVEL.

Reisch, L. A. (2005). Neue Verbraucherpolitik - Ziele, Strategien und Instrumente. Wirtschaftswissenschaftliches Studium, 36, 441-445.

Remmele, B. (2012). Macht ökonomische Bildung die Marktwirtschaft sozialer? Österreichische Zeitschrift für Soziologie, 37, 171-187.

Schrader, U. (2008). Transparenz über Corporate Social Responsibility (CSR) als Voraussetzung für einen Wandel zu nachhaltigerem Konsum. In H. Lange (Hrsg.), Nachhaltigkeit als radikaler Wandel. Die Quadratur des Kreises? (S. 149-166). Wiesbaden: VS.

Schrader, U., Liedtke, C., Lamla, J., Arens-Azevédo, U., Hagen, K., Jaquemoth, M., Kenning, P., SchmidtKessel, M., \& Strünck, C. (2013). Verbraucherpolitik für nachhaltigen Konsum - Verbraucherpolitische Perspektiven für eine nachhaltige Transformation von Wirtschaft und Gesellschaft. Stellungnahme des wissenschaftlichen Beirats Verbraucher- und Ernährungspolitik beim BMELV. https:// www.aloenk.tu-berlin.de/fileadmin/fg165/Aktuelles/Stellungnahme_Nachhaltiger_Konsum_-_final. pdf. Zugegriffen: Okt. 2016.

Steg, L. (2015). Environmental psychology and sustainable consumption. In L. A. Reisch \& J. Thøgersen (Hrsg.), Handbook of research on sustainable consumption (S. 70-83). Cheltenham: Edward Elgar.

Stiftung Warentest (STW) (2004). Sozial-ökologische Unternehmensverantwortung: Welche Maßstäbe soll man anlegen? https://www.test.de/Sozial-oekologische-Unternehmensverantwortung-WelcheMassstaebe-soll-man-anlegen-1223527-0/. Zugegriffen: Juni 2014.

Stolle, D., \& Micheletti, M. (2013). Political consumerism: Global responsibility in action. Cambridge: Cambridge University Press.

Tanner, C., \& Kast, W. (2003). Promoting sustainable consumption. Determinants of Green purchases by Swiss consumers. Psychology \& Marketing, 20, 883-902. 
Thøgersen, J. (2005). How may consumer policy empower consumers for sustainable lifestyles? Journal of Consumer Policy, 28, 143-177.

Thøgersen, J., \& Ölander, F. (2002). Human values and the emergence of a sustainable consumption pattern. A panel study. Journal of Economic Psychology, 23, 605-630.

Umweltbundesamt (2014). Umweltbewusstsein in Deutschland 2014. Ergebnisse einer repräsentativen Bevölkerungsumfrage. Berlin: Umweltbundesamt.

Verbraucherinitiative e. V. (VI) (2012). Jahresbericht 2012. Berlin: Verbraucherinitiative e. V.

Welsch, H., \& Kühling, J. (2009). Determinants of pro-environmental consumption. The role of reference groups and routine behavior. Ecological Economics, 69, 166-176.

White, H. C. (1981). Where do markets come from? American Journal of Sociology, 87, 517-547.

World Commission on Environment and Development (WCED) (1987). Our common future. Report of the World Commission on Environment and Development. http://www.un-documents.net/our-commonfuture.pdf. Zugegriffen: Okt. 2016.

Young, W., Hwang, K., McDonald, S., \& Oates, C. J. (2010). Sustainable consumption: Green consumer behaviour when purchasing products. Sustainable Development, 18, 20-31.

Zhang, X. (2015). „Voting with dollars“. A cross-polity and multilevel analysis of political consumerism. International Journal of Consumer Studies, 39, 422-436.

Sebastian Nessel geb. 1979. Dr. rer. soc. oec., Universitätsassistent am Institut für Soziologie an der KarlFranzens-Universität Graz. Forschungsschwerpunkt: Wirtschaftssoziologie. Ausgewählte Veröffentlichungen: Verbraucherorganisationen und Märkte. Eine wirtschaftssoziologische Untersuchung, 2016; Consumer organisations and the social resilience of markets, in: A. Maurer (Hrsg.), Resilience in economic and social spheres, 2016; (mit K. Kraemer, Hrsg.) Geld und Krise. Die sozialen Grundlagen moderner Geldordnungen, 2015. 